\title{
Modelo de atenção a saúde prestado no sistema carcerário feminino
}

\author{
Model of health care provided in the female prison system \\ Modelo de atención de la salud en el sistema penitenciario feminino
}

Recebido: 06/03/2021 | Revisado: 12/03/2021 | Aceito: 18/03/2021 | Publicado: 25/03/2021

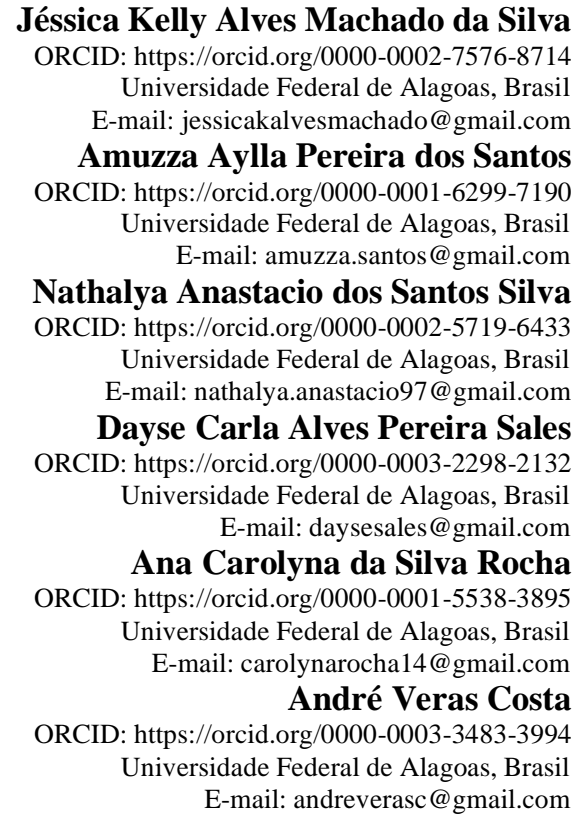

\begin{abstract}
Resumo
Objetivo: analisar o modelo de atenção a saúde prestado no atendimento às mulheres em situação de cárcere. Metodologia: estudo descritivo, exploratório com abordagem qualitativa realizado com os profissionais de saúde que atendem no Estabelecimento Prisional feminino em Alagoas. Resultados: apesar de todos os avanços ao cuidado à saúde às mulheres em situação de cárcere, predomina um modelo assistencial majoritariamente biomédico, que visa a resolução/cura de doenças e principais comorbidades apresentadas pela demanda de casos existentes. Essa realidade torna válida a discussão que modificações estruturais e na própria formação dos profissionais são necessárias para a garantia da ressocialização e reintegração social em seu mais amplo e efetivo conceito. Conclusão: um modelo preventivo no ambiente recluso e suas interferências no contexto saúde-doença contribuem para a redução de agravos, maior promoção de saúde e aumento na qualidade de vida, de tal modo que a persistência numa assistência biologicista, centrada na cura de doenças desfavorece a reintegração dessas mulheres ao convívio social.
\end{abstract}

Palavras-chave: Saúde da mulher; Enfermagem; Assistência à saúde; Prisões.

\begin{abstract}
Objective: to analyze the health care models provided in the care of women in situations of prison. Methodology: a descriptive, exploratory study with a qualitative approach carried out with the health professionals who attend the female prison in Alagoas. Results: despite all the advances in health care for women in situations of prison, a predominantly biomedical care model predominates, aimed at resolving / curing diseases and the main comorbidities presented by the demand for existing cases. This reality makes valid the discussion that structural changes and in the training of professionals are necessary to guarantee social reintegration and reintegration in its broadest and most effective concept. Conclusion: a preventive model in the recluse environment and its interference in the health-disease context contribute to the reduction of diseases, greater health promotion and increase in the quality of life, in such a way that the persistence in a biological assistance, centered on the cure of diseases, the reintegration of these women into social life.
\end{abstract}

Keywords: Women's health; Nursing; Delivery of health care; Prisons.

\section{Resumen}

Objetivo: analizar el modelo de atención de salud que se brinda a las mujeres en prisión. Metodología: estudio descriptivo, exploratorio con abordaje cualitativo realizado con los profesionales de la salud que asisten a la cárcel de mujeres de Alagoas. Resultados: apesar de todos los avances en la atención de la salud de la mujer en situación de prisión, predomina un modelo de atención predominantemente biomédico, orientado a resolver / curar las enfermedades 
y las principales comorbilidades que presenta la demanda de casos existentes. Esta realidad hace válida la discusión de que los cambios estructurales y en la formación de los profesionales son necesarios para garantizar la reinserción social y la reintegración en su concepto más amplio y efectivo. Conclusión: un modelo preventivo en el ámbito penitenciario y su injerencia en el contexto salud-enfermedad contribuyen a la reducción de enfermedades, mayor promoción de la salud y aumento de la calidad de vida, de tal manera que la persistencia en una asistencia biológica, centrada en la cura de enfermedades favorecê-la la reintegración de estas mujeres a la vida social.

Palabras clave: Salud de la mujer; Enfermería; Prestación de atención de salud; Prisiones.

\section{Introduçãa}

A criminalidade vem crescendo nos pequenos e grandes centros do mundo e os infratores são indivíduos de diferentes sexos, classe social, raça e religião, que formam uma população carcerária à mercê de um sistema punitivo, o qual contrapõe-se à reintegração social desses indivíduos (Brasil, 2014).

Em seu Plano Nacional de Saúde no Sistema Penitenciário (Brasil, 2004), o Ministério da Saúde assegura os direitos previstos na constituição, estando a saúde entre eles. Dessa forma, são disponibilizados alguns serviços através do Sistema Único de Saúde (SUS) em parceria com as Secretarias de Ressocialização. Entre eles está a Saúde da Mulher que prevê a realização de pré-natal, controle do câncer cérvico-uterino, de mama e outros agravos. Dentro desse contexto, o conjunto de ações realizadas, seja de promoção, proteção, prevenção, assistência, recuperação e vigilância em saúde, executadas nos diferentes níveis de atenção é um dos princípios da Política Nacional de Atenção Integral à Saúde das Pessoas Privadas de Liberdade no Sistema Prisional (PNAISP). Essa política tem como objetivo garantir o acesso da população privada de liberdade ao cuidado integral no SUS (Brasil, 2014).

Entretanto, um dos maiores desafios do sistema de saúde brasileiro é oferecer uma assistência que esteja orientada para a integralidade e para as necessidades ampliadas de saúde. Porém, atualmente o modelo biomédico de assistência ainda prevalece e traz problemas decorrentes dessa hegemonia. Esse cenário é destacado nas produções acadêmicas, mas na prática ainda não consegue ser detectada (Fertonani, 2015).

Quando se analisa a população carcerária feminina, observa-se que o modelo biomédico ainda prevalece em alguns contextos, no qual os fatores individual, interpessoal e ambiental (socioeconômico e político) são vistos separadamente. Assim, o ambiente prisional, necessita da identificação das características sociodemográficas e dos comportamentos de risco assumidos pelas reeducandas para que exista um entendimento desta problemática, possibilitando a utilização de modelos de assistência para o diagnóstico, seguimento, tratamento e acompanhamento dos agravos encontrados no sistema carcerário (Fertonani, 2015; Barros et al, 2016; Alves, 2007).

A partir do exposto, o presente estudo traz como pergunta norteadora "quais os modelos de atenção utilizados no atendimento às mulheres reclusas?" Nesse entendimento, o presente estudo teve como objetivo analisar o modelo de atenção a saúde prestado no atendimento às mulheres em situação de cárcere.

\section{Metodologia}

Trata-se de um estudo descritivo, exploratório com abordagem qualitativa, realizado no Estabelecimento Prisional Feminino (EPF), localizado no Estado de Alagoas, mediante solicitação de autorização para execução da pesquisa à Secretaria de Ressocialização e Inclusão Social (SERIS).

Os métodos qualitativos são aqueles nos quais é importante a interpretação por parte do pesquisador com suas opiniões sobre o fenômeno em estudo. Neles a coleta de dados muitas vezes ocorre por meio de entrevistas com questões abertas (Pereira et al., 2018). A pesquisa qualitativa, em geral, ocorre no ambiente natural com coleta direta de dados e o pesquisador é o principal instrumento. Os dados coletados são preferencialmente descritivos; a preocupação do processo é predominante em relação à do produto; o "significado" que as pessoas dão às coisas e a sua vida são focos de atenção para o pesquisador e, a análise de dados 
e informações tende a seguir um processo indutivo (Ludke \& Andre, 2013).

A amostra do estudo foi composta por todos os profissionais do bloco de saúde do estabelecimento prisional, a saber: enfermeira, assistente social, psicóloga, odontóloga e médico, além dos profissionais de nível médio, as técnicas de enfermagem.

Após a análise do serviço de saúde ofertado no EPF, a aproximação com a prática dos profissionais e leitura de revisões de literatura acerca do tema proposto, o primeiro modelo de formulário foi estruturado para ser aplicado com os profissionais de forma individual e privativa.

A coleta de dados foi realizada no período de outubro/2018 a fevereiro/2019, cumprindo em conformidade com os princípios éticos como preconiza as resoluções 466/12 e 510/16 do Conselho Nacional de Saúde, com a avaliação e o parecer do Comitê de Ética em Pesquisa da Universidade Federal de Alagoas (CEP/UFAL), sob o CAAE nº 57990816.7.0000.5013. Foi solicitado aos participantes da pesquisa a assinatura do Termo de Consentimento Livre e Esclarecido (TCLE), após esclarecimento referente ao objetivo do estudo.

O formulário semiestruturado foi o instrumento aplicado, contendo dados relacionados às variáveis de cada profissional da saúde (formação, tempo de exercício profissional, experiência profissional), à metodologia intervencionista aplicada (modelo assistencial aplicado, dados sobre as ações de saúde, resultados obtidos e metas) e sobre a dinâmica interdisciplinar entre os profissionais entrevistados (colaboração da equipe, empecilhos ligados à comunicação, e efeitos positivos obtidos a partir de abordagem interdisciplinar).

A realização da presente pesquisa respeitou as regras, escalas dos profissionais e horários disponibilizados pela unidade prisional, portanto as entrevistas foram previamente agendadas. Em virtude das escalas de serviços que os profissionais possuem, as entrevistas foram realizadas em dias e turnos diferentes, para que todos os profissionais pudessem participar do estudo. As entrevistas ocorreram nos ambientes privados de cada profissional, no qual a entrevista foi realizada seguindo o roteiro proposto, havendo a autorização para gravar a conversa.

Após a coleta, os dados foram transcritos na íntegra e organizados manualmente em um quadro contendo os questionamentos da entrevista e o perfil da assistência prestada por cada profissional. Por conseguinte, foram analisados de acordo com a técnica de Análise de Conteúdo na perspectiva de Bardin, por meio das três fases propostas pela técnica: préanálise, exploração do material, e tratamento dos resultados.

Na pré-análise foi realizada escuta e transcrição das respostas, sistematizando as ideias iniciais. A segunda fase consistiu na exploração do material através da codificação, classificação e categorização do conteúdo. Assim, foram determinados os referentes-núcleos e a divisão do texto em proposições, emergindo as categorias que representam o conteúdo. Na última fase, sucedeu-se o tratamento dos resultados, culminando nas interpretações inferenciais através da análise crítica-reflexiva.

\section{Resultados}

Observou-se que todos os profissionais, enfermeira, assistente social, psicóloga e dentista, com exceção do médico, já trabalharam em outros estabelecimentos prisionais, e por esta razão consideram que a reclusão é a principal diferença que interfere negativamente na assistência realizada, sendo assim responsável pelos notáveis agravantes à saúde, já que a privação de liberdade modifica a conduta e estratégia profissional muito específica e desafiadora, tornando-se assim um dos fatores que afronta o processo de um modelo assistencial que além de atender às demandas do sistema, promova a assistência preventiva, integral e humanizada, e consequente coparticipação no processo de ressocialização dessas mulheres reclusas.

Dessa forma, após o momento de diálogo com cada um dos profissionais envolvidos, evidenciou que apesar do pavilhão de saúde no EPF buscar um modelo assistencial de saúde, que vise as ações de promoção da saúde das reeducandas, através de recursos que fortaleçam o autocuidado, o discernimento da qualidade de saúde e uma forma integral sobre o corpo e mente dessas mulheres, a própria condição física do estabelecimento prisional, por vezes insalubre, superlotada, suscetível à contaminação e 
transmissão das mais variadas doenças, a formação mais rígida dos profissionais que exercem a segurança do estabelecimento (que por vezes, dificulta o acesso à essas reeducandas, seja por motivo de pouca escolta, seja por motivos internos do próprio ambiente), a diferença na formação e prática assistencial que cada profissional de saúde exerce, a falta de insumos essenciais para realização de exames e ações preventivas, tudo isso colabora para a falha em seguir tal modelo preventivo, tornando com isso uma assistência à saúde mais biologicista, que necessita curar rapidamente para evitar aumento nos casos de contaminação, e que é regida pelo modelo assistencial biomédico.

De tal forma, tornou-se evidente que no estabelecimento prisional a assistência prestada pelos profissionais de saúde é estruturada da seguinte forma:

\section{Metodologia intervencionista aplicada}

\section{Assistência médica}

O profissional médico do estabelecimento prisional trabalha uma vez por semana, atendendo apenas as principais demandas e agravos relacionados às especificidades clínicas. Dessa maneira, as ações assistenciais praticadas visam o diagnóstico de agravos na unidade, assim intercorre na maioria dos casos, a prescrição medicamentosa para curar os sintomas relatados pelas reeducandas, acontecendo superficialmente uma conscientização nas consultas acerca do autocuidado e bons hábitos gerais de cuidado para prevenção desses agravos. Nesse contexto, o modelo que se configura é o biomédico.

\section{Assistência de enfermagem}

A assistência de enfermagem é curativa, de caráter emergencista, porém são os profissionais de maior acesso para as reeducandas, ainda que sejam as muitas vezes atendidas após longo tempo de espera, ou em casos extremos que necessitam de cuidados, como por exemplo, picos hipertensivos, episódios de síncope, sinais de hipo ou hiperglicemia, como mais frequentes. Nesse contexto, os modelos que se configuram são o biomédico e o preventivo.

\section{Assistência psicológica}

A realidade de modelo de atendimento psicológico vigente no Estabelecimento Prisional Feminino, além de uma assistência individual e coletiva, é a inclusão de grupos terapêuticos conhecidos como grupos operativos.

Porém, durante a execução do estudo foi consolidado apenas um grupo com as gestantes do sistema prisional, funcionando como um momento em que as reeducandas ao pensar de forma coletiva, contribuem individualmente para o autocuidado, através da adoção e comprometimento de boas práticas durante a gestação. Nesse contexto, o modelo que se configura é o biopsicossocial.

\section{Assistência social}

Dentro do estabelecimento prisional, cotidianamente é prestado o apoio e atendimento assistencial às reeducandas, visando atendê-las integralmente em suas dificuldades familiares (como necessidade de contato, comunicação com os filhos e/ou parentes sobre assuntos específicos, entrega da feira, solicitação e resolução de pendências documentais, entre outros); apoio em questões financeiras; articulação com os demais serviços de saúde , apresentando possíveis problemas e dificuldades relacionadas com a atual situação de saúde de cada reeducanda; iniciando sua assistência durante a triagem (primeiro contato com os profissionais, em que ocorre a anamnese, coleta de informações e de testes de rotina do estabelecimento prisional), e sempre que necessária a obtenção de alguma informação burocrática essencial. Durante a pesquisa, tornou-se possível visualizar muito frequentemente a articulação da assistente social com diversos serviços de apoio, como por exemplo o CREAS (Centro de Referência de Assistência Social) do município da reeducanda em questão, na tentativa de achar algum parente que pudesse 
responder legalmente por ela, caracterizando uma das grandes responsabilidades de tal setor, assim como uma das grandes problemáticas que ocorre no estabelecimento prisional. Nesse contexto, o modelo que se configura é o biopsicossocial.

\section{Assistência odontológica}

Dentro da unidade prisional a assistência odontológica tem caráter de urgência, não são feitas consultas preventivas, muitas vezes por falta de materiais e insumos, anestésicos, os procedimentos são realizados sem anestésicos nos casos mais urgentes, ou até mesmo de materiais para a própria proteção dos profissionais como por exemplo, luvas, e etc.., com isso, o cuidado com a saúde bucal é deficiente, de forma que as mulheres não tem um cuidado preventivo, são atendidas na maioria das vezes em casos que não aguentam mais de dor, e esses casos resultam na exodontia, pois infelizmente não é possível reverter o quadro. Nesse contexto, o modelo que se configura é o biomédico.

\section{Discussão}

Pode-se afirmar que a população encarcerada brasileira vive abaixo da linha de dignidade mínima, não sendo a ela garantidos, malgrados declarados, seus primordiais direitos; em particular, aquele que poderia lhe conferir uma convivência saudável, no plano de um "mínimo existencial", pelo vácuo legislativo, pela dificuldade de articulação do executivo com o judiciário e no que concerne à existência e efetivação de políticas públicas baseadas nas necessidades das pessoas privadas de liberdade (Soares Filho \& Bueno, 2016).

A intensificação do encarceramento de mulheres no Brasil tem chamado atenção para diversos problemas que se relacionam às desigualdades de gênero e à necessidade de reduzir as diferentes formas de violência que se multiplicam na prisão e implicam sérios prejuízos à saúde desta população. A efetivação dos direitos reprodutivos das mulheres em situação de prisão aponta a importância de se aprofundar o debate sobre as desigualdades e violências de gênero na execução penal e nas políticas públicas voltadas para esta população (Diuana et al, 2016).

A Política Nacional de Atenção Integral à Saúde das Pessoas Privadas de Liberdade no Sistema Prisional (PNAISP) implementada pelo Ministério da Saúde através da Portaria Interministerial no 1 , de 2 de janeiro de 2014, prevê em seu Art $4^{\circ}$ que as pessoas encarceradas devem receber atenção integral e contínua visualizando qualidade da assistência nas necessidades de saúde que possam apresentar, levando em consideração as atividades preventivas e de controle de redução dos agravos (Brasil, 2014).

No entanto, à vista da realidade vivenciada durante o estudo, tornou-se possível compreender como as diversas peculiares de um ambiente recluso influenciam diretamente nas estratégias e medidas adotadas para a assistência prestada, visto que apesar da criação de metas em reuniões semanais e/ou mensais, e da ciência pelos profissionais da importância de ações de promoção à saúde e prevenção de agravos, tais peculiares, como a alta demanda proveniente das triagens, o desafio da escolta, e até o próprio manejo no atendimento, são fatores potencializadores para a falha na hegemonia de um modelo assistencial que atenda integralmente a todas as particularidades da saúde da mulher, desde as alterações em seu ciclo reprodutivo e não reprodutivo (gestação, menstruação/amenorréia, climatério/menopausa, síndromes do sistema reprodutor feminino, etc) e a todos os fatores e alterações biopsicossocioculturais, comportamentais (higiene pessoal, por exemplo), intrínsecos no processo saúde-doença dessas reeducandas, e que refletem em seu processo de ressocialização (Oliveira, 2019; Fertonani, 2015; Barros et al, 2016).

Por conseguinte, durante o estudo, tornou-se possível visualizar que ainda é vigente na assistência um modelo biomédico, que é consequência de todas as demandas e assistência prioritária, presentes no estabelecimento prisional. Com isso, a assistência à saúde dessas mulheres reclusas visa sobretudo a cura ou intervenção paliativa de tais agravos, gerados pela alta demanda (Salci et al, 2017). 
Em vista disso, tornou-se indispensável discorrer acerca do modelo seguido por cada profissional de saúde do Estabelecimento Prisional Feminino e se suas interferências no contexto saúde-doença contribuindo para a redução de agravos, maior promoção de saúde e aumento na qualidade de vida, favorecendo assim a reintegração dessas mulheres no convívio social.

\section{Assistência preventiva - Modelo biomédico}

A inadequação estrutural das prisões às necessidades específicas das mulheres - que vai desde banheiros inadequados, falta de absorventes e roupas íntimas até regulamentos que não contemplem suas especificidades - agravam as desigualdades de gênero, tornando piores as repercussões do encarceramento sobre a vida destas mulheres e de suas famílias. Muitas delas eram responsáveis pela criação dos filhos e pela manutenção da casa, e sua prisão empobrece ainda mais a família, impõe a necessidade de reorganização familiar, interrompe sua convivência com os filhos que, em muitos casos, ficam expostos a situações de desproteção. Tudo isto leva à precariedade ou mesmo à total ausência de assistência familiar à mulher presa, que fica, assim, dependente da administração prisional ou de outras presas. (Diuana et al, 2016).

De tal modo, a realidade no atendimento à saúde do Estabelecimento Prisional Feminino, envolve um estabelecimento de prioridades para a atenção às reeducandas, isto é, as mulheres com maiores fatores de risco são imediatamente encaminhadas para os profissionais, visando uma resolução dos casos. No entanto, essa forma de prestar assistência acaba funcionando como um adiamento dos cuidados, visto que um dos grandes efeitos resultantes de tal modelo, que visa apenas a cura/resolução do agravo, é que as bases desses problemas, isto é, o porquê de tais comorbidades continuarem prevalentes no ambiente prisional, não são investigadas, não ocorrendo com isso uma solução real do problema (Salci et al, 2017).

Um exemplo claro, é o tratamento medicamentoso ofertado às mulheres com algum tipo de doença crônica não transmissíveis, como diabetes e hipertensão arterial, prevalentes no sistema prisional. Ocorre que muitas reeducandas que não possuem a doença, encontram-se em situação de risco para adquirir tais patologias, em vista da alimentação fornecida no sistema, assim como fatores de risco que não são trabalhados, como o tabagismo, dentre outros (Vasconcelos et al, 2016).

No que concerne ao modelo biomédico, reconhece-se que este permanece ativo nos serviços de saúde, visando promover a resposta como forma de tratamento de diversas doenças que afligem a humanidade, predominando o foco nas intervenções individuais, as quais apenas uma parte do corpo está afetada ou não funcionante; as ações curativas predominam no tratamento das doenças, lesões e danos, tendo como aparato a medicalização e a atenção hospitalar com uso intensivo tecnologias. Pode-se mencionar, ainda, que a análise dos determinantes do processo saúde-doença, não influenciam para determinação do processo, pois o distanciamento dos aspectos que implicam nas escolhas e vivências dos sujeitos, promovem a incapacidade de compreender a multidimensionalidade do ser humano (Fertonani et al, 2015; Alves, 2007).

Por essa razão, irrefutavelmente, o modelo prestado não favorece a promoção e saúde, visando uma melhoria na qualidade de vida e uma educação em saúde, que são fatores imprescindíveis para a ressocialização efetiva dessas mulheres.

Nesse contexto, olhando para a realidade da população encarcerada, observou-se a existência de três principais modelos de assistência, sendo eles: modelo biomédico, modelo preventivo e modelo biopsicossocial de atenção à saúde. A PNAISP garante que os profissionais de saúde tenham a autonomia para executar a assistência de forma integral a todas as pessoas privadas de liberdade (Brasil, 2014).

Diante disso, reconhecendo que apesar da assistência que visa apenas a cura ou tratamento paliativo das diversas situações de saúde apresentadas por estas reeducandas (modelo biomédico), existe um grande alcance das outras vertentes de atenção citadas, caracterizadas principalmente através de uma abordagem terapêutica preventiva, analisada principalmente pela assistência de toda equipe de enfermagem, através de ações educativas e conscientização nas próprias consultas, favorecendo todo o processo de educação em saúde e contribuindo para as atividades propostas durante a pesquisa; assim como as intervenções presentes no modelo biopsicossocial, promovidas pela psicóloga e assistente social, tornando a mulher promotora 
de sua saúde e bem-estar geral, assim como intervindo significativamente desde os casos mais resolutivos aos mais severos (Farias et al, 2020).

A Política Nacional de Atenção Básica (PNAB), implementada pela Ministério da Saúde (Brasil, 2012), e revisada pela Portaria $\mathrm{n}^{\circ} 2.436$, de 21 de setembro de 2017, considera em seu artigo V como atribuições comuns a todos os profissionais de atenção básica a garantia de atenção à saúde a todos que necessitam, visando integralidade por meio de ações que promovam proteção e recuperação de agravos que possam causar danos à saúde (Brasil, 2017).

Contudo, a privação de liberdade torna a abordagem e estratégia profissional muito específica e desafiadora. Com isso, considera-se que o principal obstáculo encarado pelas mulheres para a garantia de acesso à saúde corresponde à alta demanda de reeducandas, já com agravos e/ou comorbidades, tornando-se dessa forma prioritárias no atendimento à saúde, somado à escolta escassa que é uma realidade do sistema prisional em questão. Com isso, não há a possibilidade de atendimento a todas as mulheres, não havendo o pilar essencial para a assistência à saúde, a prevenção, exacerbando ainda mais a hegemonia do modelo biomédico (Geremia, 2020).

A PNAB (Brasil 2012, 2017) determina ainda como características do processo de trabalho das equipes de atenção básica o desenvolvimento de ações que priorizem os grupos vulneráveis, realizando acolhimento, escuta qualificada e resolutividade para todos que necessitem de atendimento.

\section{Assistência preventiva e de promoção à saúde}

Um dos principais meios adotados para a prestação de uma assistência integral e que atende às particularidades de cada mulher, ocorre durante as consultas, onde a enfermeira fazendo uso de uma visão sistematizada consegue intervir antecipadamente nas mínimas mudanças e/ou queixas relatadas pelas reeducandas, assim como a prática de estimular a importância do autocuidado (Brasil, 2014).

Mesmo quando não há a possibilidade um atendimento sistematizado à todas as reeducandas, visto que a realidade ainda é uma prevenção coletiva, a enfermeira consegue ter uma comunicação efetiva com as representantes de cada cela, ficando dessa forma ciente de todos os possíveis fatores de risco de nível individual e coletivo, como as doenças transmissíveis principalmente, e traçar estratégias que atuem antecipadamente prevenindo os possíveis agravos à saúde dessas mulheres (Terra \& Campos, 2019).

Com relação à assistência psicológica, no estabelecimento há a tentativa de inserção da Terapia CognitivoComportamental (TCC), que tem por base a hipótese de que um transtorno emocional é resultado de uma vulnerabilidade cognitiva, isto é, que todas as respostas emocionais e comportamentais, são resultado da forma de como ocorre as representações de eventos internos e externos, para cada pessoa Com isso, ao inserir a prática da TCC nas mulheres reclusas a assistência consegue por meio da adequação da realidade vivida por cada reeducanda, estabelecer quais fatores internos e/ou externos são causa de transtornos psicoemocionais, e sabendo-se da causa facilitará a escolha da intervenção adequada (Capelas \& Oliveira, 2018).

Apesar de sua formação e da adoção de um modelo de atendimento biopsicossocial, ou seja, que faz a integralização de diversos fatores, enxergando as especificidades de cada indivíduo, a profissional afirma que umas das grandes diferenças na assistência à saúde no ambiente recluso, é o exercício de uma psicologia mais clínica, priorizando dessa forma os aspectos psíquicos e transtornos mentais presentes, do que uma psicologia social, que seria uma forma mais adequada às mulheres em vulnerabilidade e reclusão, visto que atende à todas vertentes que formam um ser social, como a forma de pensar e se relacionar com outras pessoas, possibilitando assim o autoconhecimento dos problemas enfrentados por essas mulheres, e uma possível mudança comportamental. 
Contudo, levando-se em consideração a dificuldade para uma assistência mais coletiva e formação de grupos operativos que atendam integralmente todas as mulheres, devido à alta demanda e pouca escolta, prevalece na assistência psicológica o atendimento sistêmico e individual, que ocorre quinzenalmente com os casos mais graves, devido à alta demanda das reeducandas provenientes da triagem, tornando-se assim uma assistência que por mais que efetiva, não alcança todas as reeducandas e principalmente os casos que com uma intervenção prévia não levariam a agravos mais severos.

\section{Processo saúde-doença sob a ótica da equipe multiprofissional}

No que concerne à comunicação inter-profissional, diariamente ocorre uma reunião com todos os profissionais de saúde presentes para tratar dos casos mais específicos, como as intercorrências ocorridas no fim do dia anterior, visando solucioná-las e sendo uma comunicação considerada eficiente por todos os profissionais entrevistados. Outra dinâmica que ocorre no bloco de saúde é o encaminhamento em demanda espontânea quando um profissional percebe que tal situação exige uma abordagem mais específica e direcionada de outro(a) profissional.

Com isso, a realidade do presídio é que por mais que haja metas que visem uma assistência mais preventiva e de promoção à saúde, a alta demanda de agravos que poderiam ter sido evitados, através de um modelo assistencial preventivo, torna-se prioridade no atendimento, estabelecendo assim um ciclo de agravo-medidas paliativas (geralmente medicamentosas) que não evolui.

A assistência prestada pela equipe multiprofissional visa a questão da otimização da consulta, de maneira que é combinada pelas profissionais o atendimento a um grupo de reeducandas diária, nas quais as mesmas passam pela consulta com a enfermeira, assistente social e psicóloga, para que assim sejam atendidas as necessidades de forma mais ampla possível, bem como, otimização do atendimento por parte da equipe multiprofissional (Farias et al, 2020).

\section{Considerações Finais}

A assistência prestada no sistema prisional ainda se encontra dividida entre ações preventivas e curativas. No entanto, ainda prevalece o modelo biomédico, com as ações curativas, por consequência dos fatores ligados ao próprio ambiente, como a grande demanda de atendimentos.

As ações de educação em saúde são elemento crucial para a prática de um modelo assistencial preventivo, principalmente na redução dos agravos em saúde, porém ele tem sido aplicado, na maioria das vezes, coletivamente, e não individualmente, podendo assim causar impactos diferentes do esperado e ajudar no controle de agravos que comprometem a saúde das pessoas que vivem em situação de cárcere.

Torna-se ainda essencial que profissionais e estudantes da área de saúde, visualizem mais essa população carcerária que é tão marginalizada, exercendo não apenas seu papel social, mas seu dever enquanto profissional de saúde sem discriminar, atendendo a todos em sua particularidade, sob uma visão holística do cuidado, através da propagação de informação e a elaboração de estudos relevantes para o compartilhamento de diferentes realidades no cárcere brasileiro, objetivando a propagação dos diferentes modelos assistenciais existentes e sua interferência não apenas no processo saúde-doença de pessoas reclusas, assim como fator determinante para que a ressocialização e reintegração social seja de fato uma realidade.

\section{Referências}

Barros, M. A. R., Cavalcanti, S. D. C., Galiza, D. D F., \& Machado, A. L. G. (2016). Situação socioeconômica e reprodutiva de mulheres presidiárias. Rev Fund Care Online, 8 (4), 4980-4985.

Brasil, Ministério da Saúde. (2004). Plano Nacional de Saúde no Sistema Penitenciário. Portaria Interministerial no 1.777 de 09 de novembro de 2003. Brasília; DF, 1-64. 
Research, Society and Development, v. 10, n. 3, e53610313619, 2021

(CC BY 4.0) | ISSN 2525-3409 | DOI: hittp://dx.doi.org/10.33448/rsd-v10i3.13619

Brasil, Ministério da Saúde. (2012). Política Nacional de Atenção Básica / Ministério da Saúde. Secretaria de Atenção à Saúde. Departamento de Atenção Básica. Brasília, 1-114.

Brasil, Ministério da Saúde. (2014). Portaria Interministerial $n^{o} 1$, de 2 de janeiro de 2014. Institui a Política Nacional de Atenção Integral à Saúde das Pessoas Privadas de Liberdade no Sistema Prisional (PNAISP) no âmbito do Sistema Único de Saúde (SUS). Diário Oficial da União, 2014.

Brasil, Ministério da Saúde. (2017). Portaria $n^{o}$ 2.436, de 21 de setembro de 2017. Aprova a Política Nacional de Atenção Básica, estabelecendo a revisão de diretrizes para a organização da Atenção Básica, no âmbito do Sistema Único de Saúde (SUS). Diário Oficial da União.

Capelas, B. E. \& Oliveira, F. M. (2018). Inovação tecnológica em saúde: o centro de atenção psicossocial como transformação do modelo assistencial. Argumentum (Vitória), 10 (1), 180-197.

Diuana, V. Ventura, M., Simas, L., Larouzé, B. \& Correia, M. (2016). Direitos reprodutivos das mulheres no sistema penitenciário: tensões e desafios na transformação da realidade. Cien Saude Colet, 21 (7), 2041-2050.

Farias, J. M \& Minghelli, L. C. \& Soratto, J. (2020). Promoção da saúde: discursos e concepções na atenção primária à saúde. Cad. saúde colet, 28 (3), 381-389.

Fertonani, H. P., Pires, D. E. P. \& Biff, D. \& Scherer, M. D. A. (2015) Modelo assistencial em saúde: conceitos e desafios para a atenção básica brasileira. Ciência e Saúde Coletiva, 20 (6), 1869-1878.

Geremia, D. S. (2020). Atenção Primária à Saúde em alerta: desafios da continuidade do modelo assistencial. Physis, 30 (1), 1-3.

Ludke, M. \& Andre, M. E. D. A. (2013). Pesquisa em educação: uma abordagem qualitativa. (2a ed.), EPU.

Oliveira, K. R. et al. (2019). Comportamentos de saúde nas experiências sexuais de mulheres em situação de cárcere. Revista Brasileira de Enfermagem, 72 (3), $88-95$.

Pereira, A. S. et al. (2018). Metodologia da pesquisa científica. UFSM.

Salci, M. A. Meirelles, B. H. S. \& Silva, D. M. G. V. (2017). Atenção primária às pessoas com diabetes mellitus na perspectiva do modelo de atenção às condições crônicas. Rev. Latino-Am. Enfermagem, 25 (2882), 1-8.

Soares Filho, M. M. \& Bueno, P. M. M. G. (2016). Demography, vulnerabilities and right to health to Brazilian prison population. Cien Saude Colet, 21 (7), 1999-2010.

Terra, L. S. V. \& Campos, G. W. S. (2019). Alienação do trabalho médico: tensões sobre o modelo biomédico e o gerencialismo na atenção primária. Trabalho, Educação e Saúde, 17 (2), 1-19.

Vasconcelos, A. C. F., Stedefeldt, E. \& Frutuoso, M. F. P. (2016). Uma experiência de integração ensino-serviço e a mudança de práticas profissionais: com a palavra, os profissionais de saúde. Interface, 20 (56), 147-158. 